This is the author's final, peer-reviewed manuscript as accepted for publication. The publisher-formatted version may be available through the publisher's web site or your institution's library.

\title{
Numerical implementation of the MRC method for obstacle scattering problems
}

Semion Gutman and Alexander G. Ramm

\section{How to cite this manuscript}

If you make reference to this version of the manuscript, use the following information:

Gutman, S., \& Ramm, A. G. (2002). Numerical implementation of the MRC method for obstacle scattering problems. Retrieved from http://krex.ksu.edu

\section{Published Version Information}

Citation: Gutman, S., \& Ramm, A. G. (2002). Numerical implementation of the MRC method for obstacle scattering problems. Journal of Physics A: Mathematical and General, 35(38), 8065-8074.

Copyright: (C) 2002 IOP Publishing Ltd

Digital Object Identifier (DOI): doi:10.1088/0305-4470/35/38/309

Publisher's Link: http://iopscience.iop.org/0305-4470/35/38/309/

This item was retrieved from the K-State Research Exchange (K-REx), the institutional repository of Kansas State University. K-REx is available at http://krex.ksu.edu 
J.Phys A, 35, (2002),8065-8074.

\title{
NUMERICAL IMPLEMENTATION OF THE MRC METHOD FOR OBSTACLE SCATTERING PROBLEMS
}

\author{
SEMION GUTMAN AND ALEXANDER G. RAMM
}

\begin{abstract}
The goal of this work is to show that the numerical solution of the obstacle scattering problem based on the MRC (modified Rayleigh conjecture) method is a competitive alternative to the BIEM (boundary integral equations method), and that it has numerical advantages which may be especially important in three-dimensional scattering problems with non-smooth domains, for example, with domains whose boundaries contain corners. MRC is formulated, the algorithm, based on it, is described, and numerical results are presented.
\end{abstract}

Key words and phrases: obstacle scattering, modified Rayleigh conjecture, numerical solution of obstacle scattering problem.

\section{INTRODUCTION}

The goal of this work is to show that the numerical solution of the obstacle scattering problem based on the MRC (modified Rayleigh conjecture) method is a competitive alternative to the BIEM (boundary integral equations method), and that it has numerical advantages which may be especially important in three-dimensional scattering problems with non-smooth domains, for example, with domains whose boundaries contain corners. Several numerical examples illustrate the above conclusions. In Section 1 the statement of the problem is formulated in the two-dimensional case. In Section 2 the MRC is formulated (see [8]), and its extension (Theorem 2.2) convenient for the numerical realization is given. In Section 3 an algorithm, based on MRC, for solving the direct scattering problem is given. In Section 4 the results of the numerical experiments are presented.

We start with the formulation of the obstacle scattering problem. In this paper we consider the Dirichlet boundary condition, but the method we develop can be used for the Neumann and Robin boundary conditions.

Let an obstacle be a bounded domain $D \subset \mathbb{R}^{2}$ with a Lipschitz boundary $\Gamma$. Fix a frequency $k>0$ and denote the exterior domain by $D^{\prime}=\mathbb{R}^{2} \backslash \bar{D}$.

A solution $v(x)$ of the Helmholtz equation

$$
\Delta v+k^{2} v=0, \quad x \in D^{\prime},
$$

is called outgoing if it satisfies the Sommerfeld radiation condition

$$
\lim _{|x| \rightarrow \infty} \sqrt{|x|}\left(\frac{\partial v}{\partial|x|}-i k v\right)=0,
$$

where the limit is attained uniformly for all the directions $x /|x|, x \in \mathbb{R}^{2}$.

The Exterior Dirichlet Problem consists of finding an outgoing solution of the Helmholtz equation (1.1) which satisfies the boundary condition

$$
v=f, \quad x \in \Gamma,
$$

where $f$ is a continuous function, see [6] for the existence and uniqueness results for this problem.

1991 Mathematics Subject Classification. Primary 35R30; PACS 43.20 Fn, 02.30 Zz, 02.60.-x . 
A particular case of the above problem is the Direct Acoustic Obstacle Scattering Problem. Let $\alpha \in S^{1}$, and the incident field be

$$
u^{i}(x)=e^{i k x \cdot \alpha}
$$

The problem is to find the total field

$$
u(x, k)=u^{i}+u^{s}, \quad x \in D^{\prime}
$$

such that

$$
u=0, \quad x \in \Gamma,
$$

and the scattered field $u^{s}$ satisfies (1.1)-(1.2).

It is known (see e.g. [6]), that every outgoing solution $v(x), x \in D^{\prime}$ has an asymptotic representation

$$
v(x)=\frac{e^{i k|x|}}{\sqrt{|x|}}\left\{A\left(\alpha^{\prime}\right)+O\left(\frac{1}{|x|}\right)\right\}, \quad|x| \rightarrow \infty,
$$

where $\alpha^{\prime}=x /|x|, \alpha^{\prime} \in S^{1}$. The function $A\left(\alpha^{\prime}\right):=A_{v}\left(\alpha^{\prime}\right)$ is called the Far Field Pattern of $u$.

For the Direct Acoustic Obstacle Scattering Problem this representation takes the form

$$
u^{s}(x)=\frac{e^{i k|x|}}{\sqrt{|x|}}\left\{A\left(\alpha^{\prime}, \alpha\right)+O\left(\frac{1}{|x|}\right)\right\}, \quad|x| \rightarrow \infty,
$$

where the uniquely defined function $A\left(\alpha^{\prime}, \alpha\right)$ is called the Scattering Amplitude of the Obstacle Scattering Problem.

Let $J_{l}(t)$ and $Y_{l}(t)$ be the Bessel and Neumann functions of the integer order $l$. The first Hankel function of order $l$ is defined by $H_{l}^{(1)}=J_{l}+i N_{l}$. Suppose that the circle $B_{R}=\left\{x \in \mathbb{R}^{2}:|x| \leq R\right\}$ contains $D$. Then, in the region $|x|>R$, the outgoing solution of the Exterior Dirichlet Problem (1.1)-(1.3) has a unique representation

$$
v(x)=\sum_{l=-\infty}^{\infty} a_{l} H_{l}^{(1)}(k|x|) e^{i l \theta}
$$

where $x /|x|=(\cos (\theta), \sin (\theta))$.

The Rayleigh Conjecture (RC) states that the series (1.9) converges up to the boundary $\Gamma$. This conjecture is false, see [1], [4], [6]. Recently A.G. Ramm [8] established a Modified Rayleigh Conjecture (MRC). In this paper the MRC approach is demonstrated to be a viable alternative for computational Direct Scattering problems. In [8] a method for solving the inverse obstacle scattering problem is also proposed. We plan to work on its numerical implementation in the near future. In [7] multidimensional inverse scattering problems are studied.

\section{Modified Rayleigh Conjecture (MRC).}

A 2-D version of the main result from [8] is

Theorem 2.1. Let (1.9) be the unique representation of the outgoing solution $v(x)$ of the Exterior Dirichlet Problem (1.1)-(1.3). Fix an $\epsilon>0$.

Then there exists a positive integer $L=L(\epsilon)$ and the coefficients $c_{l}=c_{l}(\epsilon), l=0, \pm 1, \ldots, \pm L$ such that

(i).

$$
\left\|f(x)-\sum_{l=-L}^{L} c_{l} H_{l}^{(1)}(k|x|) e^{i l \theta}\right\|_{L^{2}(\Gamma)} \leq \epsilon
$$


(ii).

where

$$
\left\|v(x)-\sum_{l=-L}^{L} c_{l} H_{l}^{(1)}(k|x|) e^{i l \theta}\right\|=O(\epsilon), \epsilon \rightarrow 0
$$

$$
\|\cdot\|=\|\cdot\|_{H_{l o c}^{m}\left(D^{\prime}\right)}+\|\cdot\|_{L^{2}\left(D^{\prime} ;(1+|x|)^{-\gamma}\right)},
$$

(iii).

$\gamma>1, m>0$ is an arbitrary integer, $H^{m}$ is the Sobolev space, and

$$
c_{l}(\epsilon) \rightarrow a_{l} \text {, as } \epsilon \rightarrow 0, l=0, \pm 1, \pm 2, \ldots .
$$

Proof. (i). Without loss of generality we can assume that the origin is an interior point of the domain $D$. Therefore there exists $\delta>0$ such that $B_{\delta}=\left\{x \in \mathbb{R}^{2}:|x|<\delta\right\} \cap \Gamma=\emptyset$, where $\Gamma$ is the boundary of $D$. Suppose that a parametric equation of $\Gamma$ is

$$
\mathbf{r}(t)=<x_{1}(t), x_{2}(t)>, \quad 0 \leq t<2 \pi .
$$

Let $r(t)=|\mathbf{r}(t)|$. Following [6], it is enough to show that functions

$$
\psi_{l}(t)=\left.H_{l}^{(1)}(k|x|) e^{i l \theta}\right|_{\Gamma}=H_{l}^{(1)}(k|r(t)|) e^{i l \theta(t)}, \quad 0 \leq t<2 \pi,
$$

where $e^{i \theta(t)}=\mathbf{r}(t) / r(t)$, and $l=0, \pm 1, \pm 2 \ldots$, form a complete system in $L^{2}(\Gamma)$. Here and in the sequel unit vectors in $\mathbb{R}^{2}$ and the corresponding complex numbers are identified as needed.

Suppose that there exists $g \in L^{2}(\Gamma)$ such that

$$
\int_{0}^{2 \pi} g(t) \psi_{l}(t) d t=0, \quad l=0, \pm 1, \pm 2, \ldots
$$

By the Addition Theorem for any $x, y \in \mathbb{R}^{2}$ with $|x|>|y|$ we have

$$
H_{0}^{(1)}(k|x-y|)=\sum_{l=-\infty}^{\infty} H_{l}^{(1)}(k|x|) e^{i l \theta_{x}} J_{l}(k|y|) e^{-i l \theta_{y}},
$$

where $x /|x|=e^{i \theta_{x}}$ and $y /|y|=e^{i \theta_{y}}$.

Recalling that the fundamental solution to the Helmholtz equation in two dimentions is

$$
\Phi(x, y)=\frac{i}{4} H_{0}^{(1)}(k|x-y|), \quad x \neq y,
$$

define the single-layer potential of $g$ by

$$
\begin{gathered}
w(y)=\frac{i}{4} \int_{\Gamma} H_{0}^{(1)}(k|x-y|) g(x) d s(x)= \\
=\frac{i}{4} \sum_{l=-\infty}^{\infty} J_{l}(k|y|) e^{-i l \theta_{y}} \int_{0}^{2 \pi} g(t) \psi_{l}(t) d t, \quad y \in B_{\delta} .
\end{gathered}
$$

Thus $w(y)=0$ for any $y \in B_{\delta}$. By the unique continuation property, $w(y)$ is identically equal to zero in $D$. The continuity of the single-layer potential implies that $w(y)=0, y \in \Gamma$. Since $w(y)$ is an outgoing solution of the Helmholtz equation in $D^{\prime}$, vanishing on $\Gamma$, it follows that $w(y)=0, y \in D^{\prime}$. Finally, the jump properties of the normal derivative of the single-layer potential imply that $g=0$ in $L^{2}(\Gamma)$.

(ii). Let the coefficients $c_{l}=c_{l}(\epsilon)$ be chosen according to (i). Denote

$$
w_{\epsilon}(x)=v(x)-\sum_{l=-L}^{L} c_{l} H_{l}^{(1)}(k|x|) e^{i l \theta} .
$$


According to Green's formula

$$
w_{\epsilon}(x)=-\int_{\Gamma} \frac{\partial \Phi(x, y)}{\partial \nu(y)} w_{\epsilon}(y) d s(y), \quad x \in D^{\prime} .
$$

Let $D \subset B_{R}=\left\{x \in \mathbb{R}^{2}:|x|<R\right\}$, and $S_{R}$ be the boundary of $B_{R}$. Since

$$
\left\|w_{\epsilon}\right\|_{L^{2}(\Gamma)}<\epsilon
$$

and

it follows that

$$
\left|\frac{\partial \Phi(x, y)}{\partial \nu(y)}\right| \leq \frac{c}{1+\sqrt{|x|}}, \quad|x| \geq R
$$

$$
\left\|w_{\epsilon}\right\|_{L^{2}\left(S_{R}\right)}<c \epsilon
$$

and

$$
\left|w_{\epsilon}(x)\right|<c \epsilon(1+\sqrt{|x|})^{-1}, \quad|x| \geq R .
$$

Therefore $w_{\epsilon}$ is $O(\epsilon)$ in the norm $L^{2}\left(D^{\prime} ;(1+\sqrt{|x|})^{-\gamma}\right)$ for $\gamma>1$.

Let $D_{R}^{\prime}=B_{R} \backslash D$. Choose $R$ such that $k^{2}$ is not a Dirichlet eigenvalue of $-\Delta$ in $D_{R}^{\prime}$. Then $w_{\epsilon}$ satisfies the following elliptic estimate from ([3], p. 189)

$$
\left\|w_{\epsilon}\right\|_{H^{m}\left(D_{R}^{\prime}\right)} \leq c\left[\left\|\left(\Delta+k^{2}\right) w_{\epsilon}\right\|_{H^{m-2}\left(D_{R}^{\prime}\right)}+\left\|w_{\epsilon}\right\|_{H^{m-1 / 2}\left(S_{R}\right)}+\left\|w_{\epsilon}\right\|_{H^{m-1 / 2}(\Gamma)}\right] .
$$

Since $\left(\Delta+k^{2}\right) w_{\epsilon}=0$ in $D^{\prime}$ this formula with $m=1 / 2$ together with (2.9) and (2.10) gives

$$
\left\|w_{\epsilon}\right\|_{H^{1 / 2}\left(D_{R}^{\prime}\right)} \leq c \epsilon .
$$

¿From local elliptic estimates and (2.13) it follows that (ii) of Theorem 2.1 holds with any $m$.

(iii). Let $x \in S_{R}$. By (2.7)

$$
w_{\epsilon}(x)=\sum_{l=-L}^{L}\left(a_{l}-c_{l}\right) H_{l}^{(1)}(k R) e^{i l \theta}+\sum_{l>|L|} a_{l} H_{l}^{(1)}(k R) e^{i l \theta} .
$$

Bessel's inequality and (2.10) imply

$$
\left|a_{l}-c_{l}\right| \leq\left\|w_{\epsilon}\right\|_{L^{2}\left(S_{R}\right)}<c \epsilon,
$$

and (iii) follows.

According to Theorem 2.1 the computation of the outgoing solution of (1.1)-(1.3) is reduced to the approximation of the boundary values in (1.3) by functions $\psi_{l}(t)$. A direct implementation of the above algorithm is efficient for domains $D$ not very different from a circle, e.g. for an ellipse with a small eccentricity (see numerical experiments in the next Section). The numerical difficulties happen because the Neumann functions $N_{l}$ with large values of $l$ are bigger than $N_{l}$ with small values of $l$ by many orders of magnitude. A finite precision of numerical computations makes it necessary to keep the values of $L$ not high, e.g. $L \leq 20$. This restriction can be remedied by adding to the set $\left\{\psi_{l}\right\}$ similar functions, centered at other interior points $x_{j} \in D$. Such an approach is justified by the following Theorem:

Theorem 2.2. Let $v(x)$ be the outgoing solution of the Exterior Dirichlet Problem (1.1)-(1.3). Suppose that points $x_{1}, x_{2}, \ldots, x_{J}$ are in the interior of $D$, and $\epsilon>0$.

Then 
(i). There exists a positive integer $L=L(\epsilon)$ and the coefficients $c_{l j}=c_{l j}(\epsilon), l=0, \pm 1, \ldots, \pm L$, $j=1,2, \ldots, J$ such that

$$
\left\|f(x)-\sum_{j=1}^{J} \sum_{l=-L}^{L} c_{l j} H_{l}^{(1)}\left(k\left|x-x_{j}\right|\right) e^{i l \theta_{j}}\right\|_{L^{2}(\Gamma)} \leq \epsilon,
$$

where $\left(x-x_{j}\right) /\left|x-x_{j}\right|=e^{i \theta_{j}}$.

(ii). Let

$$
v_{\epsilon}(x)=\sum_{j=1}^{J} \sum_{l=-L}^{L} c_{l j} H_{l}^{(1)}\left(k\left|x-x_{j}\right|\right) e^{i l \theta_{j}}
$$

\section{then}

$$
\left\|v(x)-v_{\epsilon}(x)\right\|=O(\epsilon), \epsilon \rightarrow 0
$$

where

$$
\|\cdot\|=\|\cdot\|_{H_{l o c}^{m}\left(D^{\prime}\right)}+\|\cdot\|_{L^{2}\left(D^{\prime} ;(1+|x|)^{-\gamma}\right)}
$$

$\gamma>1, m>0$ is an arbitrary integer, $H^{m}$ is the Sobolev space

(iii). The far field pattern of the approximate solution $v_{\epsilon}(x)$ is given by

$$
\begin{aligned}
& \qquad A_{v_{\epsilon}}\left(\alpha^{\prime}\right)=\sqrt{\frac{2}{\pi k}} e^{-i \frac{\pi}{4}} \sum_{j=1}^{J}\left(e^{-i k \alpha^{\prime} \cdot x_{j}} \sum_{l=-L}^{L} c_{l j}(-i)^{l} e^{i l \theta}\right), \\
& \text { where } \alpha^{\prime}=x /|x|=e^{i \theta} \text {. }
\end{aligned}
$$

Proof. Items (i) and (ii) follow from Theorem 2.1. For item (iii) note that

$$
H_{n}^{(1)}(t)=\sqrt{\frac{2}{\pi t}} e^{i(t-\pi n / 2-\pi / 4)}\left\{1+O\left(\frac{1}{t}\right)\right\}, \quad t \rightarrow \infty
$$

and

$$
\left|x-x_{j}\right|=|x|-\alpha^{\prime} \cdot x_{j}+O\left(\frac{1}{|x|}\right), \quad|x| \rightarrow \infty .
$$

Therefore

$$
H_{l}^{(1)}\left(k\left|x-x_{j}\right|\right)=\frac{e^{i k|x|}}{\sqrt{|x|}}\left\{\sqrt{\frac{2}{\pi k}} e^{i\left(-k \alpha^{\prime} \cdot x_{j}-\pi l / 2-\pi / 4\right)}+O\left(\frac{1}{|x|}\right)\right\}, \quad|x| \rightarrow \infty .
$$

Finally,

$$
e^{i \theta_{j}}=\frac{x-x_{j}}{\left|x-x_{j}\right|}=\alpha^{\prime}+O\left(\frac{1}{|x|}\right), \quad|x| \rightarrow \infty .
$$

\section{Direct Scattering Problem via MrC.}

According to Theorem 2.2 one can approximate the scattered field $u^{s}$ of the Direct Obstacle Scattering problem (1.4)-(1.6) by minimizing (2.15) with $f(x)=-u^{i}(x), x \in \Gamma$. More precisely, the algorithm can be described as follows:

Initialization. Fix an integer $L>0$ and an $\epsilon>0$. Choose $x_{1}, x_{2}, \ldots, x_{J}$ in the interior of $D$. If $\mathbf{r}(t), 0 \leq t<2 \pi$ is an equation of the boundary $\Gamma$, let

$$
\begin{gathered}
\psi_{l j}(t)=H_{l}^{(1)}\left(k\left|\mathbf{r}(t)-x_{j}\right|\right) e^{i l \theta_{j}(t)}, \quad j=1,2, \ldots, J, \quad l=0, \pm 1, \pm 2, \ldots, \pm L, \\
\left.g(t)=-u^{i}(\mathbf{r}(t))\right)=-e^{i k \mathbf{r}(t) \cdot \alpha},
\end{gathered}
$$


where $\left(\mathbf{r}(t)-x_{j}\right) /\left|\mathbf{r}(t)-x_{j}\right|=e^{i \theta_{j}(t)}$.

Minimization. Minimize

$$
\Phi(\mathbf{c})=\left\|g(t)-\sum_{j=1}^{J} \sum_{l=-L}^{L} c_{l j} \psi_{l j}(t)\right\|_{L^{2}(0,2 \pi)}, \quad \mathbf{c}=\left\{c_{l j}\right\}
$$

for $\mathbf{c} \in \mathbb{C}^{N}, N=(2 L+1) J$.

If the minimum of $\Phi$ in (3.3) is smaller than the prescribed tolerance $\epsilon$, then the scattered field is approximated by $v_{\epsilon}(x), x \in D^{\prime}$, given by (2.16), and its Scattering Amplitude $A\left(\alpha^{\prime}, \alpha\right)$ is computed by formula (2.17).

Numerical experiments also show that it may be beneficial to complement the set of functions $\psi_{l j}$ by other known outgoing solutions in $D^{\prime}$.

An example of such a solution $v(x)$ is the field scattered by a sphere $B_{a}\left(x_{0}\right) \subset D$ of radius $a>0$, subjected to the same incident wave $u^{i}(x)=e^{i k \alpha \cdot x}$ as in the original Direct Scattering problem. It is given by

$$
v(x)=-e^{i k \alpha \cdot x_{0}} \sum_{l=-\infty}^{\infty} i^{l} \frac{J_{l}(k a)}{H_{l}^{(1)}(k a)} H_{l}^{(1)}\left(k\left|x-x_{0}\right|\right) e^{i l\left(\theta_{0}-\beta\right)},
$$

and, because $\theta_{0} \rightarrow \theta$ as $|x| \rightarrow \infty, x /|x|=\alpha^{\prime}$, its scattering amplitude is

$$
A_{v}\left(\alpha^{\prime}, \alpha\right)=-\sqrt{\frac{2}{\pi k}} e^{-i \frac{\pi}{4}} e^{i k\left(\alpha-\alpha^{\prime}\right) \cdot x_{0}} \sum_{l=-\infty}^{\infty} \frac{J_{l}(k a)}{H_{l}^{(1)}(k a)} e^{i l(\theta-\beta)},
$$

where $\alpha^{\prime}=x /|x|=e^{i \theta}, \alpha=e^{i \beta}$, and $\left(x-x_{0}\right) /\left|x-x_{0}\right|=e^{i \theta_{0}}$.

The numerical implementation of the minimization algorithm begins with the choice of $M$ knots $0=t_{1}<t_{2}<\cdots<t_{M}<2 \pi$, and points $x_{j}, j=1, \ldots, J$ in the interior of $D$. Then the values $\left\{\psi_{l j}\left(t_{m}\right)\right\}_{m=1}^{M}$ form $N=(2 L+1) J$ vectors $\mathbf{a}^{(n)}, n=1,2, \ldots, N$ of length $M$. Let $\mathbf{b}=\left\{-u^{i}\left(t_{m}\right)\right\}_{m=1}^{M}$. Then the minimization problem (3.3) is reduced to the finite dimensional minimization problem

$$
\min \left\{\|A \mathbf{c}-\mathbf{b}\|, \quad \mathbf{c} \in \mathbb{C}^{N}\right\}
$$

where $A$ is the matrix containing vectors $\mathbf{a}^{(n)}, n=1,2, \ldots, N$ as its columns. If other outgoing solutions are used in addition to functions $\psi_{l j}$, the size of matrix $A$ is increased accordingly.

We use the Singular Value Decomposition (SVD) method (see e.g. [5]) to minimize (3.6). Small singular values of the matrix $A$ are used to identify and delete linearly dependent or almost linearly dependent combinations of vectors $\mathbf{a}^{(n)}$. This spectral cut-off makes the minimization process stable. The entire algorithm is summarized below. We denote by $V^{H}$ the complex conjugate transpose of a matrix $V$. Also, by the definition, the inner product in $\mathbb{C}^{N}$ complex conjugates its first component.

Iterative MRC. Fix an $\epsilon>0$, an integer $L>0$, and $w_{\min }>0$.

Choose $M$ knots $0=t_{1}<t_{2}<\cdots<t_{M}<2 \pi$, and points $x_{j}, j=1, \ldots, J$ in the interior of $D$. Let $N=(2 L+1) J$.

(1) Initialization.

(a) Form vectors

$$
\mathbf{a}^{(n)}=\left\{\psi_{l j}\left(t_{m}\right)\right\}_{m=1}^{M}, \quad|l| \leq L, \quad j=1,2, \ldots, J,
$$

and the matrix $A$ of size $M \times N$, whose columns are the vectors $\mathbf{a}^{(n)}$.

(b) Form vector

$$
\mathbf{b}=\left\{-u^{i}\left(t_{m}\right)\right\}_{m=1}^{M}
$$


(c) Use Singular Value Decomposition method to represent matrix $A$ as

$$
A=U W V^{H} \text {, }
$$

where the $M \times N$ matrix $U$ has orthonormal columns $\mathbf{u}^{(n)}, n=1, \ldots, N$, the square $N \times N$ matrix $V$ has orthonormal columns $\mathbf{v}^{(n)}, n=1, \ldots, N$, and the diagonal square $N \times N$ matrix $W=\left(w_{n}\right)_{n=1}^{N}$ is composed of the (nonnegative) singular values of $A$.

(d) Let $\Sigma \subset\{1,2, \ldots, N\}$ be defined by

$$
\Sigma=\left\{n: w_{n} \geq w_{\min }\right\} \text {. }
$$

(e) Let $p=0$.

(2) Iterative step.

(a) Let $p:=p+1$.

(b) Form the set

$$
P=\left\{n \in \Sigma: w_{n} \text { is among } p \text { largest singular values of } A\right\} .
$$

(c) Compute the normalized residual

$$
r_{p}^{\min }=\frac{1}{\sqrt{M}} \sqrt{\|\mathbf{b}\|^{2}-\sum_{n \in P}\left|<\mathbf{u}^{(n)}, \mathbf{b}>\right|^{2}} .
$$

(3) Stopping criterion.

(a) If $r_{p}^{\text {min }} \leq \epsilon$, then stop.

The minimizer is given by

$$
\mathbf{c}=\sum_{n \in P} \frac{1}{w_{n}}<\mathbf{u}^{(n)}, \mathbf{b}>\mathbf{v}^{(n)}
$$

Compute the scattered field $v_{\epsilon}^{s}$ using (2.16) and the Far Field Pattern using (2.17).

(b) If $r_{p}^{\text {min }}>\epsilon$, and $P \neq \Sigma$ repeat the iterative step (2).

(c) Otherwise, the procedure has failed.

\section{Numerical Experiments.}

The results obtained by the MRC method (for smooth boundary $\Gamma$ ) were compared to the results obtained by the Boundary Integral Equation Method (BIEM) as presented in [2]. Accordingly, to find the outgoing solution $v(x)$ of the Exterior Dirichlet Problem (1.1)-(1.3) one has to solve the integral equation

$$
\varphi(x)+\int_{\Gamma}\left\{\frac{\partial \Phi(x, y)}{\partial \nu(y)}-i \eta \Phi(x . y)\right\} \varphi(y) d s(y)=2 f(x), \quad x \in D^{\prime}
$$

for the density function $\varphi \in C(\Gamma)$. Following the recommendations in [2] the value for the real coupling parameter $\eta$ was chosen to be equal to the wave number $k$. The above integral equation was solved using the Nyström method, see [2], Section 3.5.

After the density $\varphi$ is computed, the Far Field Pattern can be obtained from

$$
A_{v}\left(\alpha^{\prime}\right)=\frac{e^{-i \frac{\pi}{4}}}{\sqrt{8 \pi k}} \int_{\Gamma}\left\{k \nu(y) \cdot \alpha^{\prime}+\eta\right\} e^{-i k \alpha^{\prime} \cdot y} \varphi(y) d s(y),
$$

where $\nu$ is the exterior unit normal vector to the boundary $\Gamma$.

We conducted numerical experiments for four obstacles: two ellipses of different eccentricity, a kite, and a triangle. Each case was tested for wave numbers $k=1.0$ and $k=5.0$. Each obstacle was subjected to incident waves corresponding to $\alpha=(1.0,0.0)$ and $\alpha=(0.0,1.0)$. The results are shown in Table 1 . The column $J$ shows the number of the interior points $x_{j}$ used in the 
TABLE 1. Normalized residuals attained in the numerical experiments.

\begin{tabular}{crrcrr}
\hline Experiment & $J$ & $k$ & $\alpha$ & $r^{\text {min }}$ & $\hat{r}^{\text {min }}$ \\
\hline I & 4 & 1.0 & $(1.0,0.0)$ & 0.000201 & 0.000096 \\
& 4 & 1.0 & $(0.0,1.0)$ & 0.000357 & 0.000324 \\
& 4 & 5.0 & $(1.0,0.0)$ & 0.001309 & 0.000556 \\
& 4 & 5.0 & $(0.0,1.0)$ & 0.007228 & 0.002156 \\
\hline II & 16 & 1.0 & $(1.0,0.0)$ & 0.003555 & 0.003555 \\
& 16 & 1.0 & $(0.0,1.0)$ & 0.002169 & 0.002169 \\
& 16 & 5.0 & $(1.0,0.0)$ & 0.009673 & 0.009286 \\
& 16 & 5.0 & $(0.0,1.0)$ & 0.007291 & 0.007056 \\
\hline III & 16 & 1.0 & $(1.0,0.0)$ & 0.008281 & 0.008281 \\
& 16 & 1.0 & $(0.0,1.0)$ & 0.007523 & 0.007523 \\
& 16 & 5.0 & $(1.0,0.0)$ & 0.021571 & 0.021530 \\
& 16 & 5.0 & $(0.0,1.0)$ & 0.024360 & 0.024206 \\
\hline IV & 32 & 1.0 & $(1.0,0.0)$ & 0.006610 & 0.006610 \\
& 32 & 1.0 & $(0.0,1.0)$ & 0.006785 & 0.006785 \\
& 32 & 5.0 & $(1.0,0.0)$ & 0.034027 & 0.033218 \\
& 32 & 5.0 & $(0.0,1.0)$ & 0.040129 & 0.038558 \\
\hline
\end{tabular}

approximation (3.3). The choice of the points $x_{j}$ was different in each experiment. It is indicated below together with the description of the experiments. The column $r^{\text {min }}$ shows the smallest value of the normalized residual achieved by the MRC minimization method in step 2.c of the Iterative MRC algorithm described in the previous section.

We also tested the algorithm with the outgoing solutions (3.4) induced by spheres in $D$. These scattering solutions were used in addition to functions $\psi_{l j}$. The spheres were centered at the same interior points $x_{j}$ chosen in the experiment. The radius of each of the spheres is chosen to be the biggest one that fits it within the domain $D$. Thus we allow the spheres to intersect each other, but still be entirely inside the domain $D$. The column with $\hat{r}^{\text {min }}$ shows the values of the normalized residual attained in this version of the MRC algorithm. Values $L=5$ and $M=720$ were used in all the experiments. The 720 knots $t_{m}$ were uniformly distributed on $[0,2 \pi]$.

Table 2 shows the scattering amplitude for vectors $\alpha^{\prime}$ at every multiple of $30^{\circ}$ for Experiment I, and the ratio (denoted by MRC/BIEM) of the scattering amplitude computed by the MRC method and by the BIEM. The BIEM was implemented as described in the beginning of this section with $n=64$ (see [2]). The scattering amplitudes shown correspond to the last column in Table 1.

Experiment I. The boundary $\Gamma$ is an ellipse described by

$$
\mathbf{r}(t)=(2.0 \cos t, \sin t), \quad 0 \leq t<2 \pi .
$$

The MRC minimization used 4 interior points $x_{j}=0.7 \mathbf{r}\left(\frac{\pi(j-1)}{2}\right), j=1, \ldots, 4$. Run time for the MRC was 2 seconds vs. 25 seconds for the BIEM on a $333 \mathrm{MHz} \mathrm{PC}$.

Experiment II. The kite-shaped boundary $\Gamma$ (see [2], Section 3.5) is described by

$$
\mathbf{r}(t)=(-0.65+\cos t+0.65 \cos 2 t, 1.5 \sin t), \quad 0 \leq t<2 \pi,
$$

see Figure 1. The MRC minimization used 16 interior points $x_{j}=0.9 \mathbf{r}\left(\frac{\pi(j-1)}{8}\right), j=1, \ldots, 16$. Run time for the MRC was 33 seconds vs. 44 seconds for the BIEM.

Experiment III. The boundary $\Gamma$ is the triangle with vertices at $(-1.0,0.0)$ and $(1.0, \pm 1.0)$. The MRC minimization used 16 interior points $x_{j}=0.9 \mathbf{r}\left(\frac{\pi(j-1)}{8}\right), j=1, \ldots, 16$. Run time for 
Table 2. Scattering Amplitude $A\left(\alpha^{\prime}, \alpha\right)$ in Experiment I for $\alpha=(1.0,0.0)$ and $k=5.0$.

\begin{tabular}{rrr}
\hline$\alpha^{\prime}$ & $A\left(\alpha^{\prime}, \alpha\right)$ & MRC/BIEM \\
\hline $0^{\circ}$ & $-0.016258-0.642831 i$ & $1.000115+0.000082 i$ \\
$30^{\circ}$ & $0.413163+0.337149 i$ & $1.000213-0.000035 i$ \\
$60^{\circ}$ & $0.273417-0.621033 i$ & $0.999987+0.000300 i$ \\
$90^{\circ}$ & $-3.000307+2.365251 i$ & $0.999990-0.000084 i$ \\
$120^{\circ}$ & $0.273418-0.621033 i$ & $0.999912+0.000406 i$ \\
$150^{\circ}$ & $0.413163+0.337149 i$ & $1.000135-0.000022 i$ \\
$180^{\circ}$ & $-0.016258-0.642831 i$ & $1.000001-0.000001 i$ \\
$210^{\circ}$ & $-0.341318-0.799098 i$ & $1.000041-0.000014 i$ \\
$240^{\circ}$ & $0.482573-1.109062 i$ & $0.999997-0.000034 i$ \\
$270^{\circ}$ & $1.176199-0.783961 i$ & $0.999960-0.000041 i$ \\
$300^{\circ}$ & $0.482572-1.109062 i$ & $1.000039-0.000093 i$ \\
$330^{\circ}$ & $-0.341318-0.799098 i$ & $1.000002+0.000016 i$ \\
\hline
\end{tabular}

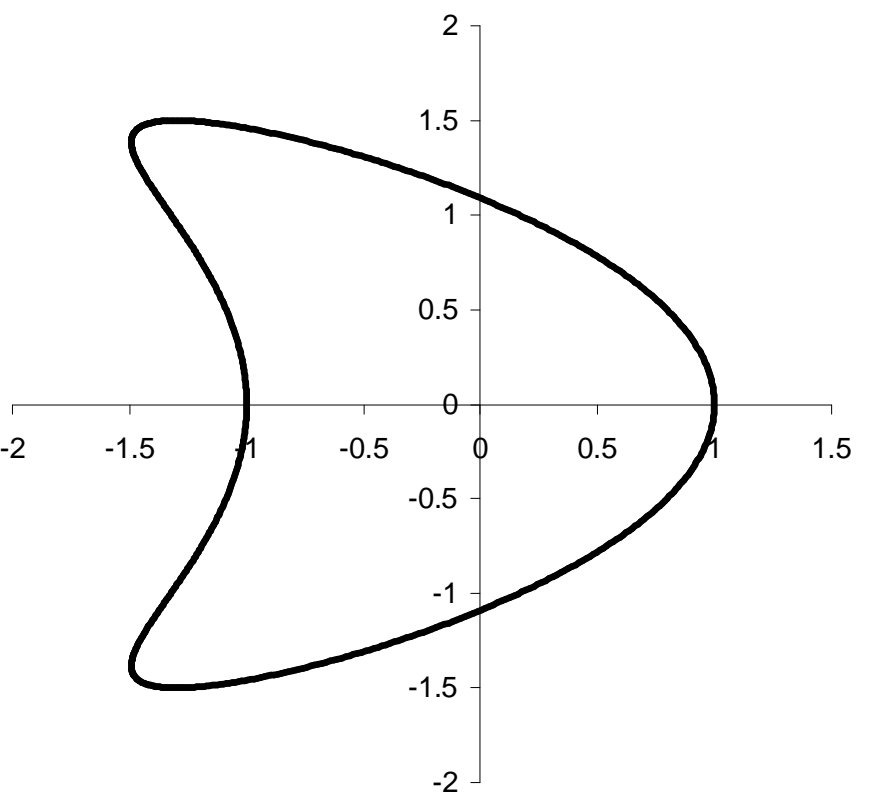

Figure 1. The kite-shaped obstacle used in Experiment II.

the MRC was about 30 seconds. See Figure 2 for the scattering amplitude $A\left(\alpha^{\prime}, \alpha\right)$ for $\alpha=$ $(0.0,1.0), k=5.0$ obtained by the MRC method with these parameters.

Experiment IV. The boundary $\Gamma$ is an ellipse described by

$$
\mathbf{r}(t)=(0.1 \cos t, \sin t), \quad 0 \leq t<2 \pi .
$$




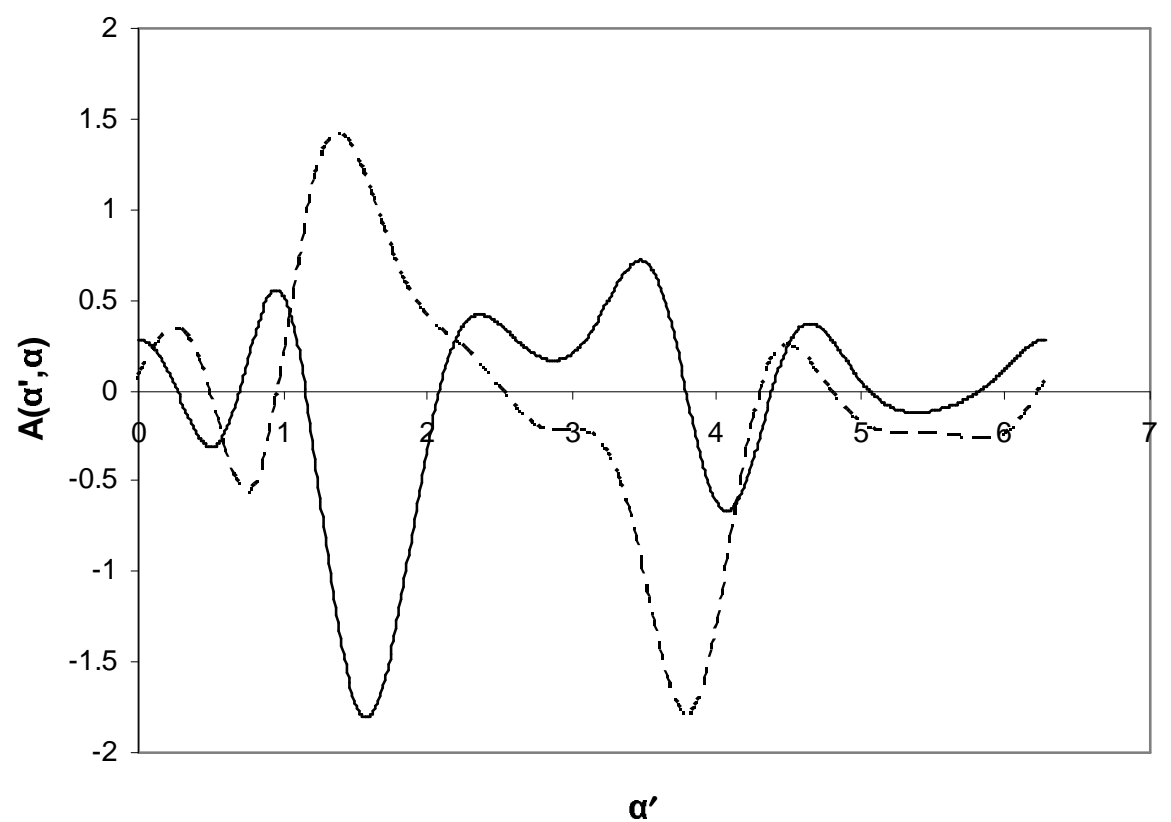

Figure 2. Real (solid line) and imaginary (dotted line) parts of the scattering amplitude $A\left(\alpha^{\prime}, \alpha\right)$ for Experiment III, $\alpha=(0.0,1.0)$ and $k=5.0$.

The MRC minimization used 32 interior points $x_{j}=0.95 \mathbf{r}\left(\frac{\pi(j-1)}{16}\right), j=1, \ldots, 32$. Run time for the MRC was about 140 seconds.

\section{Conclusions.}

Computation of scattering solutions via the Modified Rayleigh Conjecture method provides an alternative to the Boundary Integral Equation Method. BIEM representations (4.1) and (4.2) are exact, while MRC provides an approximate method. The advantages of MRC consists of its easy implementation in both 2-D and 3-D cases. Also its performance is shown to be better, or, at least, equal to the BIEM. In contrast to BIEM, no additional programming is needed for domains with corners. Additional studies are needed to fine tune the performance of the MRC algorithm, but it seems that the most dramatic improvement over the BIEM may be for 3-D obstacles. The usage of the outgoing waves, scattered by spheres, improves the algorithm, especially if their centers are located away from the boundary of the obstacle. The improvement is more pronounced for values of $L<5$. The choice of the spheres inscribed in the domain $D$ was the most efficient in the conducted experiments.

\section{REFERENCES}

[1] Barantsev R, [1971] Concerning the Rayleigh hypothesis in the problem of scattering from finite bodies of arbitrary shapes, Vestnik Lenungrad Univ., Math., Mech., Astron., 7, 56-62. 
[2] Colton D., Kress R. [1992] Inverse Acoustic and Electromagnetic Scattering Theory, Springer-Verlag, New York.

[3] Lions J.L., Magenes E. [1972] Non-Homogeneous Boundary Value Problems and Applications, Springer, New York.

[4] Millar R. [1973] The Raleigh hypothesis and a related least-squares solution to the scattering problems for periodic surfaces and other scatterers, Radio Sci., 8, 785-796.

[5] Press W.H., Teukolsky S.A., Vetterling W.T., Flannery B.P. [1992] Numerical Recepies in FORTRAN, Second Ed., Cambridge University Press.

[6] Ramm A.G. [1986] Scattering by Obstacles, D. Reidel Publishing, Dordrecht, Holland.

[7] Ramm A.G. [1992] Multidimensional Inverse Scattering Problems, Longman/Wiley, New York.

[8] Ramm A.G. [2002] Modified Rayleigh Conjecture and Applications, J. Phys. A: Math. Gen. 35, L357-L361.

Department of Mathematics, University of Oklahoma, Norman, OK 73019, USA

E-mail address: sgutman@ou.edu

Department of Mathematics, Kansas State University, Manhattan, Kansas 66506-2602, Usa

E-mail address: ramm@ath.ksu.edu 